\title{
Meningioangiomatosis. Descripción de dos casos y revisión de la literatura
}

\author{
A. Suárez-Gauthier; M.R. Gómez de la Bárcena; E. García-García; J. Hinojosa* y J.R. Ricoy
}

Departamentos de Anatomía Patológica / Neuropatología y *Neurocirugía infantil. Hospital Universitario 12 de Octubre. Madrid.

Resumen

La Meningioangiomatosis (MA) es una lesión benigna intracraneal poco frecuente. La mayoría de los casos son aislados, pero también se han descrito casos asociados a Neurofibromatosis (NF) tipo 2. Los casos asociados a NF suelen ser asintomáticos. Los casos no asociados a NF son sintomáticos, se presentan en niños $y$ adultos jóvenes, $y$ en general afectan a leptomeninge y a corteza cerebral subyacente.

En este trabajo se describen 2 casos no asociados a NF en niños de 1 y 7 años de edad, que se manifestaron por crisis, las cuales desaparecieron tras la extirpación de la lesión. Histológicamente las lesiones eran de predominio celular en un caso y de predominio fibro-hialino en el otro.

De la revisión de la literatura se concluye que las meningioangiomatosis no asociadas a NF suelen ser lesiones únicas, clínicamente suelen presentarse con crisis convulsivas, $y$ pueden verse asociadas a otras patologías, fundamentalmente a meningiomas.

Histológicamente las lesiones corresponden a una proliferación celular de hábito meningotelial que se dispone alrededor de los vasos corticales. A pesar de que todos los casos tienen características comunes, existe un amplio espectro de formas histológicas, que abarca desde casos más celulares hasta casos más fibrosos con calcificaciones. Estas diferencias podrían corresponder a diferentes estadios evolutivos de la lesión. La extirpación de la lesión conlleva la desaparición de los síntomas.

PALABRAS CLAVE: Corteza cerebral. Hamartoma, Leptomeninge. Meningioangiomatosis. Meningioma. Tumor.

Meningioangiomatosis: report of two cases and literature review

Recibido: 01-02.05. Acepatdo: 18-02-05
Summary

Meningioangiomatosis (MA) is a rare benign intracraneal lesion. The majority of cases are sporadic although the association of this lesion with familial neurofibromatosis (NF) type 2 is well known. NF-associated MA may be multifocal and is often asymptomatic and diagnosed at autopsy. Non-associated cases are usually symptomatic, occurs in children and young adults and frequently arise in leptomeninges and underlying cerebral cortex.

In the present work, we describe two new non-associated cases of MA in two boys, seven and one year old with seizures that disappeared after surgical excision.

Histopathologically, the lesion was predominantly cellular in one case and more fibrous in the other.

From the literature review we concluded that sporadic cases present as single lesions which manifest by seizures or persistent headaches. Rarely MA has been described to coexist with meningiomas.

Histopathologically, MA is characterized by a plaque-like proliferation of meningothelial and fibroblast-like cells surrounding small vessels and trapping islands of gliotic cortical tissue. The lesion does not show significant atypia, mitosis or necrosis. Although all cases of MA share unifying features, there are different degrees of histological presentation with cases predominantly cellular and others more fibrous and calcified. This could correspond to different stages in the evolution of the MA. Symptoms disappear with the complete excision of the lesion.

KEY WORDS: Cerebral cortex. Hamartoma. Leptomeninge. Meningioangiomatosis. Meningioma. Tumour.

\section{Introducción}

La meningioangiomatosis (MA) es una lesión intracraneal benigna poco frecuente, que suele presentarse en niños

Abreviaturas. MA: meningioangiomatosis. NF: neurofibromatosis 
y adultos jóvenes. Clínicamente puede ser asintomático o presentar desde dolor de cabeza hasta crisis convulsivas.

Se han descrito casos aislados y casos asociados a neurofibromatosis (NF) tipo 2 y a meningiomas (con o $\sin$ NF tipo 2). En los pacientes con NF la MA es, en general, clínicamente silente, representando un hallazgo incidental en la autopsia ${ }^{34}$. Wiebe y cols. ${ }^{34}$ aportaron 7 nuevos casos de MA no asociada a NF. En su trabajo analizaron 13 casos publicados de MA asociados a NF y 43 no asociados a NF (dos de ellos publicados por uno de nosotros ${ }^{9}$.

Posteriormente al estudio de Wiebe y cols. ${ }^{34}$, se han publicado 27 casos nuevos no asociados a NF, lo que totaliza 77 hasta ahora descritos.

En este trabajo describimos dos nuevos casos no asociados a NF, y revisamos los aspectos clínicos y patológicos más relevantes de la MA no asociada a NF 2.

\section{Casos clínicos}

Caso 1. Varón de 7 años, nervioso e hiperquinético, que durante 2 o 3 meses presentó episodios nocturnos de sobresalto, de los cuales se despertaba llorando o hablando en voz alta sobre episodios vividos durante el día.

La exploración neurológica sólo mostró una dudosa afectación campimétrica temporal superior izquierda del ojo izquierdo.

En el TC craneal se apreció una imagen calcificada densa témporo-occipital derecha, con patrón en girasol, sin realce después de la inyección de contraste, con atrofia focal del parénquima subyacente. Con RM se observó una masa hipointensa de 4'5 × 3'5 × 2'5cm, sin carácter infiltrativo.

Se realizó una craniectomía occipital derecha, y se procedió a la extirpación microquirúrgica de la lesión.

Histológicamente, en el espesor de la corteza cerebral se aprecia un nódulo constituido por abundante tejido hialinizado, con depósitos de sales cálcicas e islotes de células fusiformes dispuestas en haces, sin evidencia de tejido nervioso. En la periferia del nódulo se aprecian yemas de células fusiformes sin atipia que aíslan nódulos de tejido nervioso con gliosis (Figura 1). El diagnóstico anátomopatológico es de MA. Ocho años después de la intervención quirúrgica el paciente está asintomático y sin medicación.

Caso 2. Varón de 1 año de edad, que acude al hospital tras presentar un episodio que comenzó con movimientos clónicos en los dedos de la mano izquierda, que se propagaron a toda la mano y más tarde a todo el hemicuerpo izquierdo. Junto a estos, presentó parpadeo de ojo izquierdo con desviación de la mirada hacia la izquierda. La duración total del episodio fue de 15 a $20 \mathrm{~min}$.

La TC craneal mostró una imagen no calcificada cortical temporal, asociada a una zona de realce confluente con zonas lineales y puntuales, acompañado de edema

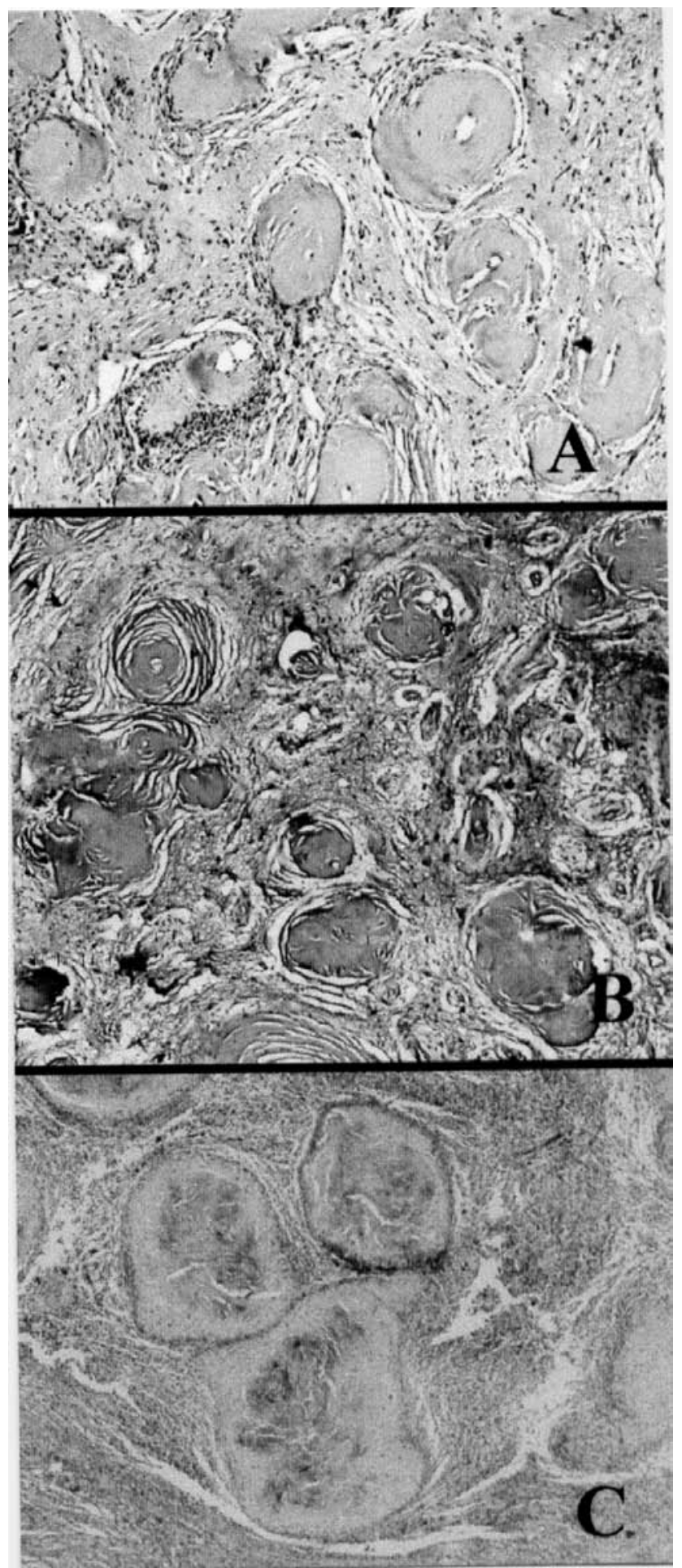

Figura 1. Caso 1: En 1. A y 1. B se observan nódulos hialinos perivasculares con escasa celularidad fusiforme en la periferia de éstos (1A: HE x 40, 1.B: Tricromico de Masson $x$ 40). En 1. C se observan nódulos hialinos con depósitos de sales cálcicas y yemas de células fusiformes en la periferia de la lesión (HE x 100). 
vasogénico. Con RM se observó una lesión córticosubcortical en la vertiente posterior de la circunvolución temporal superior derecha, de $2 \times 2 \times 2 \mathrm{~cm}$., sin provocar efecto masa significativo, multilobulada. Los componentes sólidos presentan realce significativo tras la administración de contraste intravenoso. En la sustancia blanca adyacente se apreció un cambio de señal sugerente de corresponder a ligero edema vasogénico que no produce efecto masa significativo.

Se extirpó la lesión mediante craneotomía temporal derecha.

Histológicamente la leptomeninge está engrosada por aumento de la celularidad en forma de placa. Desde la leptomeninge se proyectan hacia el interior de la corteza digitaciones de células fusiformes u ovaladas que se disponen alrededor de vasos, formando tabiques intracorticales, que aíslan nódulos de tejido nervioso (Figura 2). No se observa fibrosis, hialinización ni depósitos de sales cálcicas. El diagnóstico anatomopatológico es de MA.

Cinco meses después de la intervención quirúrgica, el paciente no presenta crisis.

\section{Discusión}

Tras el trabajo de Wiebe y cols. ${ }^{34}$ se han publicado al menos 27 pacientes con MA no asociada a NF, que, junto a los 2 aportados en este estudio totalizan 29 nuevos casos más ${ }^{1,47,12,13,15,16,18,19,21-24,28-32}$.

Un $63 \%$ de los pacientes ${ }^{50}$ son varones y un $33 \%{ }^{29}$ mujeres, observándose por tanto un claro predominio masculino.

La distribución por edad de los pacientes analizados por Wiebe y cols. ${ }^{34}$ abarcaba desde los 9 meses hasta los 70 años, con una media de 28 años de edad; en los estudios posteriores las edades abarcan desde los 12 meses hasta los 71 años, con una media de 21 años. La media de edad de los 79 casos publicados es de 24 años.

La manifestación clínica más frecuente observada en 45 de los 79 casos descritos son las convulsiones (57\%). El resto de los casos presentaron desde dolor de cabeza a dolor facial o parálisis de pares faciales. En 3 casos no hemos podido recoger datos sobre la clínica ${ }^{13,21,30}$.

Comúnmente la MA es intracortical y localizada en región fronto-temporal derecha, si bien también puede ser bi-hemisférica9 ${ }^{9}$ Se han descrito localizaciones atípicas como cuerpo calloso ${ }^{14} \mathrm{y}$ tronco cerebral ${ }^{6,17}$.

La lesión suele ser única, aunque también se han descrito lesiones múltiples, generalmente en casos asociados a $\mathrm{NF}^{5,10,11,27}$, pero también en casos aislados ${ }^{24,25}$.

Aunque se han descrito casos de MA asociados a malformaciones arterio-venosas, a encefalocele ${ }^{33}$ y a oligodendroglioma ${ }^{20}$, la asociación más frecuente es con un meningioma. En la literatura se han descrito 14 casos de

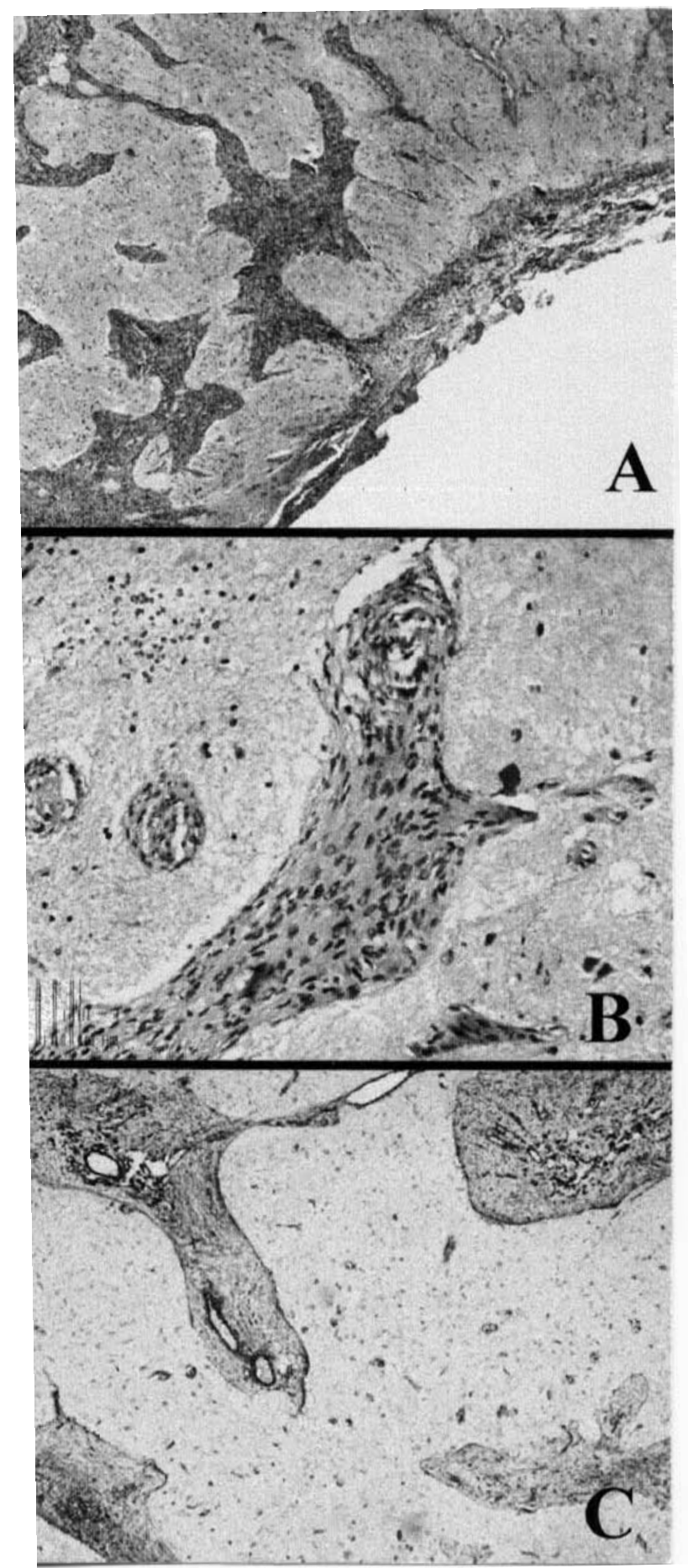

Figura 2. Caso 2: En 2. A se aprecia engrosamiento de la leptomeninge con proyecciones digitiformes penetrando hacia el interior de la corteza (HE x 40). En 2. B y 2. $C$ se observa un detalle de las digitaciones con células fusiformes alrededor de los vasos (HE x 200) En 2. C las células de las digitaciones son inmunorreactivas para Vimentina (x 100). 
MA asociada a meningioma ${ }^{2,3,7,15,19,21,30,35}$.

Histológicamente, se trata de una proliferación de células meningoteliales, inmunorreactivas para vimentina y EMA, que puede mostrarse como un engrosamiento focal de la leptomeninge desde la que penetran tabiques en la corteza, o bien como una lesión exclusivamente intracortical sin relación con la leptomeninge. La magnitud de las lesiones intracorticales es también variable, desde pequeños tabiques o fascículos perivasculares hasta auténticos nódulos.

En la literatura están descritos casos que muestran este amplio espectro histológico, desde patrones celulares ${ }^{18}$ hasta patrones fibrosos y calcificados ${ }^{12}$. Estas diferencias podrían deberse a estadios evolutivos distintos de la lesión, siendo las lesiones predominantemente fibrosas las consecutivas a la evolución de las lesiones predominantemente celulares.

De hecho, los casos presentados son diferentes histológicamente, pudiendo corresponder el Caso 2 (1 año de edad) a un estadio más inicial, con engrosamiento de la leptomeninge y tabiques constituidos por células fusiformes penetrando en la corteza, y el Caso 1 (7 años de edad), a un estadio más avanzado, con una lesión intracortical, con menos celularidad y con nódulos hialinos.

La MA es una lesión poco conocida cuya patogenia no está clara. Se ha relacionado con un hamartoma, con un meningioma e incluso con una malformación vascular ${ }^{8,17,26}$. Wiebe y $\operatorname{cols}^{34}$ sugieren el origen de la MA en una célula pluripotencial que se diferenciaría en los diferentes componentes encontrados en esta lesión. Otros autores ${ }^{12}$ hablan de un origen fibroblástico o en células de músculo liso.

El diagnóstico diferencial más importante de la MA es con el meningioma, ya que el pronóstico y evolución de estas dos entidades es completamente distinta. Además la MA puede simular un meningioma invasivo.

Típicamente se habla de meningioma invasivo cuando las células tumorales rompen la piamadre infiltrando la corteza subyacente. El patrón de crecimiento en nidos y la eventual presencia de atipia y mitosis apoyaría el diagnóstico de meningioma invasivo. En caso de ausencia de atipia celular, el patrón de crecimiento perivascular y la presencia de ovillos neurofibrilares en las neuronas del tejido cortical residual $^{10,22}$ apoyarían el diagnóstico de MA.

También se ha descrito un caso de meningioma intraparenquimatoso bien delimitado del parénquima adyacente y constituido por células que expresaban vimentina y EMA, asociado a MA adyacente con ovillos neurofibrilares en el tejido cortical respetado ${ }^{23}$. Se postuló que el meningioma se originaba a partir de la MA, aunque también podría corresponder a un nódulo hiperplásico de la MA.

En resumen, la MA sintomática suele presentarse durante la infancia y adolescencia y se manifiesta por convulsiones. Es una lesión única, cortical o cortico-subcortical, en la región frontotemporal. $\mathrm{Su}$ extirpación quirúrgica conlleva la desaparición de las crisis. Histológicamente muestra un espectro que abarca desde formas celulares a formas fibrosas, algunas hialinizadas y calcificadas. Dada su frecuente asociación a meningiomas, debe diferenciarse de meningiomas infiltrantes.

\section{Bibliografía}

1. Al-Hindi, H., Subach, B., Hamilton, R.L.: Case of the month: A 7 years old girl with seizures. Brain Pathol 1997; 7 : 1023-1024.

2. Auer, R.N., Budny, J., Drake, C.G., Ball, M.J.: Frontal Lobe perivascular schwannoma. Case report. J Neurosurg 1982; 56:154-157.

3. Blumenthal, D., Berho, M., Bloommfield, S.S., Schochet, S.S. Jr, and Kaufman, H.H.: Childhood meningioma associated with meningioangiomatosis. J Neurosurg 1993; 72: 287-289.

4. Chakrabarty, A., Franks, A.J.: Meningioangiomatosis: a case report and review of the literature. Br J Neurosurg 1999 Apr 13(2): 167-173.

5. Foerster, O., Gagel, O.: Ein Fall von Recklinghausenscher Krankheit mit funf nebeneinander bestehenden verschiedenartigen Tumorbildungen. Ztschr f d ges Neurol u Psychiat 1932; 138: 339-360.

6. Garen, P.D., Powers, J.M., King, J.S., Perot, P.L. Jr.: Intracranial fibroosseus lesion. J Neurosurg 1989; 70: 475477.

7. Giangaspero, F., Guiducci, A., Lenz, F.A., Mastronardi, L., and Burger, P.C.: Meningioma with meningioangiomatosis: a condition mimicking invasive meningiomas in children and young adults. Am J Surg Pathol 1999; 23: 872-875.

8. Goates, J.J., Dickson, D.W., Horoupian, D.S.: Meningioangiomatosis: an inmunocytochemical study. Acta Neuropathol (Berl) 1991; 82:527-532.

9. Gómez-Ansón, B., Munoz, A., Blasco, A., Madero, S., Esparza, J., Cordobés, F., et al.: Meningioangiomatosis: advanced imaging and pathological study of two cases. Neuroradiology 1995; 37:120-123.

10. Halper, J., Scheithauer, B.W., Okazaki, H., et al.: Meningioangiomatosis: a report of six cases with special references to the occurrence of neurofibrillary tangles. J Neuropathol Exp Neurol 1986; 45: 426-446.

11. Hozay, J.: Une angioneuromatose meningo-encephalique diffuse.Rev Neurol (Paris) 1953; 89: 222-236.

12. Izycka-Swieszewska Rzepko, R., Kopczynski, S., Franc, Z., Szurowska, E., Borowska, L.J.: Meningioangiomatosis with a fibrocalciying component.Neuropathology 2000; 20: 44-48.

13. Jorge, C.L., Nagahashi-Marie, S.K., Pedreira, C.C., Rosemberg, S., et al.: Clinical characteristics and surgical outcome of patients with temporal lobe tumors and epilepsy. Arq 
Neuropsiquiatr 2000; 58: 1002-1008.

14. Jun, C., Burdick, B.: An unusual fibro-osseus lesion of the brain. J Neurosurg 1984; 60: 1308-1311.

15. Kim, N.R., Choe, G., Shin, S.H., Wang, K.C., Cho, B.K. et al.: Childhood meningiomas associated with meningioangiomatosis: report of five cases and literature review. Neuropath Appl Neurob 2002; 28: 48-56.

16. Kim, W-Y., Kim, I-O., Kim, W-S., Cheon, J-E., and Yeon, K.M.: Meningioangiomatosis: MR imaging and pathological correlation in two cases. Ped Radiol 2002; 32: 96-98.

17. Kollias, S.S., Crone, K.R., Ball, W.S., Prenger, E.C., Ballard, E.T.: Meningioangiomatosis of the brain stem: case report. J Neurosurg 1994; 80: 732-735.

18. Koutsopoulos, A.V., Yannopoulos, A., Stathopoulos, E.N., Evangeliou, A., Panayiotides, J.G. et al.: Meningioangiomatosis with predominantly cellular pattern. Neuropathology 2003; 23: 141-145.

19. Kuchelmeister, K., Richter, H.P., Kepes, J.J., and Schachenmayr, W.: Case report: microcystic meningioma in a 58-year-old man with multicystic meningiangiomatosis. Neuropath Appl Neurobiol 2003; 29: 170-177.

20. López, J.I., Ereño, C., Oleaga, L., Areitio, E.: Meningioangiomatosis and oligodendroglioma in a 15-year-old boy. Arch Pathol Lab Med 1996; 120: 587-590.

21. Meyer, S., Romeike, B., Strowitzki, M., Grunewald, I., Graf, N., et al.: Meningioangiomatosis with associated meningioma in a 4-year-old girl presenting with a focal. Nevenartz 2002; 73: 990-994.

22. Mokhtari, K., Uchihara, T., Clemenceau, S., Baulac, M., Duyckaerts, C., Hauw, J.J.: Atypical neuronal inclusion bodies in meningioangiomatosis. Acta Neuropathol (Berl) 1998; 96: 91-96.

23. Mut, M., Soylemezoglu, F., Firat, M.M., Palaoglu, S.: Intraparenchymal meningioma originating from underlying meningioangiomatosis. J Neurosurg 2000; 92: 706-710.

24. Park, M.S., Suh, D.C., Choi, W.s., Lee, S.Y., and Kang, G.H.: Multifocal meningioangiomatosis: a report of two cases. Am J Neurorad 1999; 20: 677-680.

25. Paulus, W., Peiffer, J., Roggendorf, W., Schuppan, D.: Meningioangiomatosis. Pathol Res Pract 1989; 184: 446-454.

26. Prayson, R.A.: Meningioangiomatosis. A clinicopathologic study including MIB-1 inmunoreactivity. Arch Pathol
Lab 1995;119: 1061-1064.

27. Rubinstein, L.J.: Tumeurs et hamartomes dans la neurofibromatose centrale. In: Michaux, L., Feld, M., editors. Les Phakomatoses cerebrales. Paris: S.P.E.I. ; 1963 : 426-451.

28. Savargaonkar, P., Bhuiya, T., Valderrama, E., Farmer, P.: Scrape cytology of meningioangiomatosis: a report of two cases with diagnostic cytologic features. Acta Cytoly 2001; 45: 1069-1072.

29. Savargaonkar, P., Chen, S., Bhuiya, T., Valderrama, E., Bloom, T., Farmer, P.M.: Meningioangiomatosis: report of three cases and review of the literature. Ann Clin Lab Sci 2003; 33: 115-118.

30. Sinkre, P., Perry, A., Cai, D., Raghavan, R., Watson, M., Wilson, K., Barton Rogers, B.: Deletion of the NF2 region in both meningioma and yuxtaposed meningioangiomatosis: case report supporting a neoplastic relationship. Pediatric Dev Pathol 2001; 4: 568-572.

31. Tacconi, L., Thom, M., Symon, L.: Cerebral meningioangiomatosis: case report. Surg Neurol 1997; 48: 255-260.

32. Takeshima, Y., Amatya, V.J., Nakayori, F., Nakano, T., Sugiyama, K., Inai, K.: Meningioangiomatosis ocurring in a young male without neurofibromatosis: with special reference to its histogenesis and loss of heterozygosity in the NF2 gene region. Am J Surg Pathol 2002; 26: 125-129.

33. Whiting, D.M., Awad, I.A., Miles, J., Chou, S.S., Luders, H.: Intractable complex partial seizures associated with occult temporal lobe encephalocele and meningioangiomatosis: a case report. Surg Neurol 1990; 34: 318-322.

34. Wiebe, S., Muñoz, D.G., Smith, S., Lee, D.H.: Meningioangiomatosis: a comprehensive analysis of clinical and laboratory features. Brain 1999; 122: 709-726.

35. Wilson, D., Dempsey, R.J., Clark, D.B.: Meningioma developing from underlying meningioangiomatosis. J Neuropathol Exp Neurol 1991; 50: 371.

Suárez-Gauthier, A.; Gómez de la Bárcena, M.R.; GarcíaGarcía, E.; Hinojosa, J.; Ricoy, J.R.: Meningioangiomatosis. Descripción de dos casos y revisión de la literatura. Neurocirugía 2006; 17: 250-254.

Correspondencia postal: José R. Ricoy. Anatomía Patológica / Neuropatología. Hospital Universitario 12 de Octubre. Avda de Córdoba, s/n. 28041 - Madrid 